\title{
A Functional Generalized Hill Process and Its Uniform Theory
}

\author{
Gane Samb Lo $0^{1,2} \&$ El Hadji Dème ${ }^{2}$
}

${ }^{1}$ LSTA, Université Pierre et Marie Curie, France

${ }^{2}$ LERSTAD, Université Gaston Berger de Saint-Louis, Senegal

Correspondence: Gane Samb Lo, Université Gaston Berger, Saint-Louis BP 234, Senegal. Tel: 221-33-961-2340.

E-mail: gane-samb.lo@ugb.edu.sn, ganesamblo@ufrsat.org

Received: July 25, 2012 Accepted: September 19, 2012 Online Published: October 29, 2012

doi:10.5539/ijsp.v1n2p250 URL: http://dx.doi.org/10.5539/ijsp.v1n2p250

\section{Abstract}

We are concerned in this paper with the functional asymptotic behavior of the sequence of stochastic processes

$$
T_{n}(f)=\sum_{j=1}^{j=k} f(j)\left(\log X_{n-j+1, n}-\log X_{n-j, n}\right),
$$

indexed by some classes $\mathcal{F}$ of functions $f: \mathbb{N} \backslash\{0\} \longmapsto \mathbb{R}_{+}$and where $k=k(n)$ satisfies

$$
1 \leq k \leq n, k / n \rightarrow 0 \text { as } n \rightarrow \infty .
$$

This is a functional generalized Hill process including as many new estimators of the extreme value index when $F$ is in the extreme value domain. We focus in this paper on its functional and uniform asymptotic law in the new setting of weak convergence in the space of bounded real functions. The results are next particularized for explicit examples of classes $\mathcal{F}$.

Keywords: extreme values theory, asymptotic distribution, functional Gaussian and nongaussian laws, uniform entropy numbers, asymptotic tightness, stochastic process of estimators of extreme value index, sowly and regularly varying functions

2010 Mathematics Subject Classification: Primary 62E20, 62F12, 60F05; Secondary 60B10, 60F17

\section{Introduction}

Let $X_{1}, X_{2}, \ldots$ be a sequence of independent copies (s.i.c) of a real random variable (r.v.) $X>1$ with d.f. $F(x)=$ $\mathbb{P}(X \leq x)$. We will be concerned in this paper with the functional asymptotic behavior of the sequence of stochastic processes

$$
T_{n}(f)=\sum_{j=1}^{k} f(j)\left(\log X_{n-j+1, n}-\log X_{n-j, n}\right),
$$

indexed by some classes $\mathcal{F}$ of functions $f: \mathbb{N}^{*}=\mathbb{N} \backslash\{0\} \longmapsto \mathbb{R}_{+}$and where $k=k(n)$ satisfies

$$
1 \leq k \leq n, k / n \rightarrow 0 \text { as } n \rightarrow \infty .
$$

The main motivation of this study is to obtain very large classes of estimators for the extreme when $F$ lies in the extreme value domain, all of them being margins of only one stochastic process. Indeed, for the uniform function $f(j)=j, k^{-1} T_{n}(f)$ is the famous Hill (1975) estimator of such an index. Recently, a first step to functional forms of the Hill estimator has been done in Diop and Lo (2009) in the form $k^{-\tau} T_{n}(f)$ for $f(j)=j^{\tau}$ and respectively studied in Diop and Lo (2009) for $(\tau>1 / 2)$ and in Dème, LO and Diop (2010) for $(0<\tau<1 / 2)$ in finite distributions. Groenboom, Lopuha and Wolf (2003). also considered a thorough study of a family of Kernel-type estimators of the extreme value index. However, they did not consider a stochastic processes view. There exists a very large number of estimators of the extreme velue index. We may cite those of Csörgó-Deheuvels-Mason (1985), De Haan-Resnick (1980), Pickands (1975), Deckkers, De Haan and Einmahl (1989), Hasofer and Wang (1992), etc. But they all go back to the Hill's one. 
However, the asymptotic theory for the estimators of the extreme value index are set for a finite number of them, in finite distributions for whole the extreme value domain $(-\infty \leq \gamma \leq+\infty)$. The reader is referred to following sample citations: Csörgö, Deheuvels and Mason (1985); Csörgö and Mason (1985); Dekkers, Einmahl and De Haan (1989); Diop and Lo (2009); De Haan and Resnick (1980); Hall (1982); Hall (1978); Hauesler and Teugels (1985); Hasofer and Wang (1992); Lo (1989); Pickands (1975) etc.

Now the modern setting of functional weak convergence allows to handle more complex estimators in form of stochastic processes, say $\left\{T_{n}(f), f \in \mathcal{F}\right\}$, such that for any $f \in \mathcal{F}$, there exists a nonrandom sequence $a_{n}(f)$ such that $T_{n} / a_{n}(f)$ is an estimator of the extreme value index $\gamma$. Such processes may be called stochastic processes of estimators of the extreme value index.

Here, we consider one of such processes, that is (1.1). Our aim is to derive their functional asymptotic normality when possible or simply their asymptotic distribution for suitable classes. We will see that for some classes, we have non Gaussian asymptotic behavior, which will be entirely characterized. We shall mainly consider two classes of functions. The first consists of those functions $f$ satisfying

$$
A(2, f)=\sum_{j=1}^{\infty} f(j)^{2} j^{-2}<\infty,
$$

with the general notation $A(m, f)=\sum_{j=1}^{\infty} f(j)^{m} j^{-m}$. The second includes functions $f$ such that

$$
\limsup _{n \rightarrow+\infty} B(n, f)=0,
$$

where $B(n, f)=\sigma_{n}(f)^{-1} \max \left\{f(j) j^{-1}, 1 \leq j \leq k\right\}$ and $\sigma_{n}(f)$ is defined below in (1.2). Under these two conditions, we will be able to find the asymptotic distributions of $T_{n}(f)$ for a fixed $f$, under usual and classical hypotheses of extreme value Theory. But as to functional laws, we need uniform conditions. Define $\mathcal{F}_{1}$ the subclass of $\mathcal{F}$ such that

$$
0<\inf _{f \in \mathcal{F}_{1}} A(2, f)<\sup _{f \in \mathcal{F}_{1}} A(2, f)<+\infty,
$$

and $\mathcal{F}_{2}$ be a subclass of $\mathcal{F}$ such that

$$
\lim _{n \rightarrow \infty} \sup _{f \in \mathbb{F}_{2}} B(n, f)=0
$$

and such that for any couple $\left(f_{1}, f_{2}\right) \in \mathcal{F}_{2}$,

$$
\lim _{n \rightarrow \infty} \frac{1}{\sigma_{n}\left(f_{1}\right) \sigma_{n}\left(f_{2}\right)} \sum_{j=1}^{k} f_{1}(j) f_{2}(j) j^{-2}=\Gamma\left(f_{1}, f_{2}\right)
$$

exists, where

$$
\sigma_{n}^{2}(f)=\sum_{j=1}^{k} f(j)^{2} j^{-2} \text { and } a_{n}(f)=\sum_{j=1}^{k} f(j) j^{-1} .
$$

We will suppose at times that each $\mathcal{F}_{h}$ is totally bounded with respect to some semimetric $\rho_{h}$.

Our best achievement is the complete description of the weak convergence of the sequence

$$
\left\{T_{n}(f), f \in \mathcal{F}_{h}\right\}, h=1,2,
$$

in the spaces $\ell^{\infty}\left(\mathcal{F}_{h}\right)$ of bounded and real functions defined on $\mathcal{F}_{h}$, in the light of the modern setting of this theory in Theorems 3 and 4. Further we provide real case studies with explicit classes in application of the general results, in corollaries of Section 5.

This approach yields a very great number of estimators of the tail distribution $1-F$ in the extreme value domain. But, this paper will essentially focus on the functional and uniform laws of the process described above. Including in the present work, for example, second and third order conditions as it is fashion now, and considering data driven applications or simulation studies would extremely extend the report. These questions are to be considering in subsequent papers.

This paper will use technical results of extreme value theory. So we will summarize some basics of this theory in the Section 2. In this section, we introduce basic notation and usual representation of distribution functions lying 
in the extreme value domain, to be used in all the remainder of the paper. Section 3 is devoted to pointwise limit distributions of $T_{n}(f)$, while our general functional results are stated and established in Section 4 . In Section 5, we study some particular cases, especially the family $\left\{f(j)=j^{\tau}, \tau>0\right\}$. The section 6 is devoted to the tools of the paper. In this latter, we state two lemmas, namely Lemmas 1 and 2, which are key tools in the earlier proofs.

We draw the attention of the reader that we will deal with uniform converges on families of random variables indexed set functions denoted here by $\mathcal{F}_{h}$. It is not sure that the supremum of such families of random variables are measurable. So we are obliged to use the convergence in outer probability rather than convergence in probability in the case where measurability is not proved. We remind here this concept. A sequence of applications $\left(Z_{n}\right)_{n \geq 1}$ defined on $(\Omega, \mathcal{A}, \mathbb{P})$ with values in $\mathbb{R}$ converges to zero in outer probability as $n \rightarrow+\infty$ if and only there exists a sequence real and nonnegative random variables $\left(u_{n}\right)_{n \geq 1}$ defined on $(\Omega, \mathcal{A}, \mathbb{P})$ such that : $\forall n \geq 1,\left|Z_{n}\right| \leq\left|u_{n}\right|$ and $u_{n}$ tends to zero in probability as $n \rightarrow+\infty$. This is also denoted by $Z_{n}=o_{P}^{*}$. (See van der Vaart \& Wellner, 1996) for a complete account of such a theory)

\section{Some Basics of Extreme Value Theory}

The reader is referred to de Haan (1970; 2006), Resnick (1987), Galambos (1985) and Beirlant, Goegebeur and Teugels (2004) for a modern and large account of the extreme value theory. A distribution function $F$ is said to be attracted to a non degenerated $M$ iff the maximum $X_{n, n}=\max \left(X_{1}, \ldots X_{n}\right)$, when appropriately centered and normalized by two sequences of real numbers $\left(a_{n}>0\right)_{n \geq 0}$ and $\left(b_{n}\right)_{n \geq 0}$, converges to $\mathrm{M}$, in the sense that

$$
\lim _{n \rightarrow+\infty} P\left(X_{n, n} \leq a_{n} x+b_{n}\right)=\lim _{n \rightarrow+\infty} F^{n}\left(a_{n} x+b_{n}\right)=M(x),
$$

for continuity points $x$ of $M$.

If (2.1) holds, it is said that $F$ is attracted to $M$ or $F$ belongs to the domain of attraction of $M$, written $F \in D(M)$. It is well-known that the three possible nondegenerate limits in (2.1), called extreme value d.f., are the following.

The Gumbel d.f.

$$
\Lambda(x)=\exp (-\exp (-x)), x \in \mathbb{R}
$$

or the Fréchet $d . f$. of parameter $\alpha>0$,

$$
\phi_{\gamma}(x)=\exp \left(-x^{-\alpha}\right) \mathbb{I}_{[0,+\infty[}(x), x \in \mathbb{R}
$$

or the Weibull d.f. of parameter $\alpha>0$

$$
\psi_{\gamma}(x)=\exp \left(-(x)^{-\alpha}\right) \mathbb{I}_{]-\infty, 0]}(x)+\left(1-1_{]-\infty, 0]}(x)\right), \quad x \in \mathbb{R},
$$

where $\mathbb{I}_{A}$ denotes the indicator function of the set A. Now put $D(\phi)=\cup_{\alpha>0} D\left(\phi_{\gamma}\right), D(\psi)=\cup_{\alpha>0} D\left(\psi_{\gamma}\right)$, and $\Gamma=D(\phi) \cup D(\psi) \cup D(\Lambda)$.

In fact the limiting distribution function $M$ is defined by an equivalence class of the binary relation $\mathcal{R}$ on the set of d.f's $\mathcal{D}$ in $\mathbb{R}$ defined as follows :

$$
\begin{gathered}
\forall\left(M_{1}, M_{2}\right) \in \mathcal{D}^{2},\left(M_{1} \mathcal{R} M_{2}\right) \Leftrightarrow \exists(a, b) \in \mathbb{R}_{+} \backslash\{0\} \times \mathbb{R}, \forall(x \in \mathbb{R}), \\
M_{2}(x)=M_{1}(a x+b) .
\end{gathered}
$$

One easily checks that if $F^{n}\left(a_{n} x+b_{n}\right) \rightarrow M_{1}(x)$, then $F^{n}\left(c_{n} x+d_{n}\right) \rightarrow M_{1}(a x+b)=M_{2}(x)$ whenever

$$
a_{n} / d_{n} \rightarrow a \text { and }\left(b_{n}-d_{n}\right) / c_{n} \rightarrow b \text { as } n \rightarrow \infty \text {. }
$$

These facts allow to parameterize the class of extreme value distribution functions. For this purpose, suppose that (2.1) holds for the three d.f.'s given in (2.2), (2.3) and (2.4). We may take sequences $\left(a_{n}>0\right)_{n \geq 1}$ and $\left(b_{n}\right)_{n \geq 1}$ such that the limits in (2.5) are $a=\gamma=1 / \alpha$ and $b=1$ (in the case of Fréchet extreme value domain), and $a=-\gamma=-1 / \alpha$ and $b=-1$ (in the case of Weibull extreme value domain). Finally, one may interprets $(1+\gamma x)^{-1 / \gamma}=\exp (-x)$ for $\gamma=0$ (in the case of Gumbel extreme value domain). This leads to the following parameterized extreme value distribution function

$$
G_{\gamma}(x)=\exp \left(-(1+\gamma x)^{-1 / \gamma}\right), 1+\gamma x \geq 0
$$

called the Generalized Extreme Value (GEV) distribution of parameter $\gamma \in \mathbb{R}$. 
Now we give the usual representations of $d f^{\prime} s$ lying in the extreme value domain in terms of the quantile function of $\mathrm{G}(\mathrm{x})=\mathrm{F}\left(\mathrm{e}^{x}\right), x \geq 1$, that is $G^{-1}(1-u)=\log F^{-1}(1-u), 0 \leq u \leq 1$.

Theorem 1 We have:

\section{1) Karamata's representation (KARARE)}

(a) If $F \in D\left(\phi_{1 / \gamma}\right), \gamma>0$, then

$$
G^{-1}(1-u)=\log c+\log (1+p(u))-\gamma \log u+\left(\int_{u}^{1} b(t) t^{-1} d t\right), 0<u<1,
$$

where $\sup (|p(u)|,|b(u)|) \rightarrow 0$ as $u \rightarrow 0$ and $c$ is a positive constant and $G^{-1}(1-u)=\inf \{x, G(x) \geq u\}, 0 \leq u \leq 1$, is the generalized inverse of $G$ with $G^{-1}(0)=G^{-1}(0+)$.

(b) If $F \in D\left(\psi_{1 / \gamma}\right), \gamma>0$, then $y_{0}(G)=\sup \{x, G(x)<1\}<+\infty$ and

$$
y_{0}-G^{-1}(1-u)=c(1+p(u)) u^{\gamma} \exp \left(\int_{u}^{1} b(t) t^{-1} d t\right), 0<u<1,
$$

where $c, p(\cdot)$ and $b(\cdot)$ are as in (2.7).

2) Representation of de Haan (Theorem 2.4.1, 1970),

If $G \in D(\Lambda)$, then

$$
G^{-1}(1-u)=d-s(u)+\int_{u}^{1} s(t) t^{-1} d t, 0<u<1,
$$

where $d$ is a constant and $s(\cdot)$ admits this KARARE:

$$
s(u)=c(1+p(u)) \exp \left(\int_{u}^{1} b(t) t^{-1} d t\right), 0<u<1,
$$

c, $p(\cdot)$ anf $b(\cdot)$ being defined as in (2.7).

We restrict ourselves here to the cases $F \in D(\Gamma) \cup D\left(\Phi_{1 / \gamma}\right), \gamma>0$, since the case $F \in D\left(\Psi_{1 / \gamma}\right), \gamma>0$, may be studied through the transform $\left.F\left(x_{0}(F)-1 /\right)\right) \in D\left(\Phi_{1 / \gamma}\right)$ for estimating $\gamma$. This leads to replacle $X_{n-j+1, n}$ by $x_{0}(F)-1 / 1 / X_{j, n}$ in (1.1). However, a direct investigation of (1.1) for $F \in D\left(\Psi_{1 / \gamma}\right), \gamma>0$ is possible. This requires the theory of sums of dependent random variables while this paper uses results on sums of independent random variables, as it will be seen shortly. We consequently consider a special handling of this case in a distinct paper.

Finally, we shall also use the uniform representation of $Y_{1}=\log X_{1}, Y_{2}=\log X_{2}, \ldots$ by $G^{-1}\left(1-U_{1}\right), G^{-1}\left(1-U_{2}\right), \ldots$ where $U_{1}, U_{2}, \ldots$ are independent and uniform random variables on $(0,1)$ and where $G$ is the $d$.f. of $Y$, in the sense of equality in distribution (denoted by $=_{d}$ )

$$
\left\{Y_{j}, j \geq 1\right\}={ }_{d}\left\{G^{-1}\left(1-U_{j}\right), j \geq 1\right\}
$$

and hence

$$
\begin{gathered}
\left\{\left\{Y_{1, n}, Y_{2, n}, \ldots Y_{n, n}\right\}, n \geq 1\right\} \\
={ }_{d}\left\{\left\{G^{-1}\left(1-U_{n, n}\right), G^{-1}\left(1-U_{n-1, n}\right), \ldots, G^{-1}\left(1-U_{1, n}\right)\right\}, n \geq 1\right\} .
\end{gathered}
$$

In connection with this, we shall use the following Malmquist representation (see Shorack \& Wellner, 1986 , p. 336) :

$$
\left\{\log \left(\frac{U_{j+1, n}}{U_{j, n}}\right)^{j}, j=1, \ldots, n\right\}={ }_{d}\left\{E_{1}, \ldots, E_{n}\right\},
$$

where $E_{1}, \ldots, E_{n}$ are independent standard exponential random variables.

\section{Pointwise and Finite-distribution Laws of $T_{n}(f)$}

Let us begin to introduce these conditions on the distribution function $G$, through the functions $p$ and $b$ in the representations (2.7), (2.8), (2.9) and (2.10). First, define for $\lambda>1$,

$$
0 \leq g_{1, n}(p, \lambda)=\sup _{0 \leq u \leq \lambda k / n}|p(u)|,
$$




$$
g_{2, n}(b, \lambda)=\sup _{0 \leq u \leq \lambda k / n}|b(u)|,
$$

and

$$
d_{n}(p, b, \lambda)=\max \left(g_{1, n}(p, \lambda), g_{2, n}(b, \lambda) \log k\right) .
$$

We will need the following conditions for some $\lambda>1$ :

$$
\begin{gathered}
d_{n}(p, b, \lambda) \rightarrow 0 \text { as } n \rightarrow \infty . \\
g_{1, n}(f, \lambda)\left(\sigma_{n}(f)\right)^{-1} \sum_{j=1}^{k} f(j) \rightarrow 0 \text {, as } n \rightarrow \infty, \\
g_{2, n}(b, \lambda)\left(\sigma_{n}(\tau) k^{\tau}\right)^{-1} \sum_{j=1}^{k} f(j) \rightarrow 0 \text { as } n \rightarrow \infty
\end{gathered}
$$

and

$$
d_{n}(p, b, \lambda)\left(\sigma_{n}(f) k^{\tau}\right)^{-1} \sum_{j=1}^{k} f(j) \rightarrow 0 \text { as } n \rightarrow \infty .
$$

From now on, all the limits are meant as $n \rightarrow \infty$ unless the contrary is explicitly stated. We are able to state:

Theorem 2 Let $F \in D(\Lambda)$. If $(C 0)$ and $(C 3)$ holds, then

$$
\left(s(k / n) \sigma_{n}(f)\right)^{-1}\left(T_{n}(f)-a_{n}(f) s(k / n)\right) \rightarrow \mathcal{N}(0,1)
$$

when (K2) holds and

$$
\left(s(k / n) \sigma_{n}(f)\right)^{-1}\left(T_{n}(f)-a_{n}(f) s(k / n)\right) \rightarrow \mathcal{L}(f),
$$

when (K1) is satisfied, and where

$$
\mathcal{L}(f)=A(2, f)^{-1 / 2} \sum_{j=1}^{\infty} f(j) j^{-1}\left(E_{j}-1\right)
$$

Let $F \in D\left(\phi_{1 / \gamma}\right)$. If $(C 1)$ and $(C 2)$ hold, then

$$
\left(a_{n}(f) / \sigma_{n}(f)\right)\left(T_{n}(f) / a_{n}(f)-\gamma\right) \rightarrow \mathcal{N}\left(0, \gamma^{2}\right)
$$

under (K2) and

$$
\left(a_{n}(f) / \sigma_{n}(f)\right)\left(T_{n}(f) / a_{n}(f)-\gamma\right) \rightarrow \gamma^{-1} \mathcal{L}(f)
$$

under $(K 1)$.

Proof. Let us use the representation (2.11). We thus have, for any $n \geq 1$,

$$
\left\{\log X_{n-j+1, n}=Y_{n-j+1, n}, 1 \leq j \leq n\right\}={ }_{d}\left\{G^{-1}\left\{1-U_{j, n}\right), 1 \leq j \leq n\right\} .
$$

First, let $F \in D(\Lambda)$. By (2.8), we get

$$
T_{n}(f)=\sum_{j=1}^{k} f(j)\left(s\left(U_{j, n}\right)-s\left(U_{j+1, n}\right)\right)+\sum_{j=1}^{k} f(j) \int_{U_{j, n}}^{U_{j+1, n}} s(t) / t d t \equiv S_{n}(1)+S_{n}(2) .
$$

Using (2.10), we have for $U_{1, n} \leq v, u \leq U_{k, n}$,

$$
s(u) / s(v)=(1+p(u)) /(1+p(v)) \exp \left(-\int_{U_{1, n}}^{U_{k+1, n}} t^{-1} b(t) d t\right) .
$$

Putting

$$
g_{1, n, 0}(p)=\sup \left\{|p(u)|, 0 \leq u \leq U_{k+1, n}\right\} \text { and } g_{2, n, 0}(p)=\sup \left\{|b(u)|, 0 \leq u \leq U_{k+1, n}\right\}
$$


we get, since $\log \left(U_{k+1, n} / U_{1, n}\right)=O_{p}(\log k)$ as $n \rightarrow \infty$,

$$
s(u) / s(v)=\left(1+O\left(g_{1, n, 0}\right)\right) \exp \left(-O_{p}\left(g_{2, n, 0} \log k\right)\right) .
$$

This implies

$$
\sup _{U_{1, n} \leq u, v \leq U_{k, n}}|s(u) / s(v)-1|=O_{p}\left(\max \left(g_{1, n, 0}, g_{2, n, 0} \log k\right)\right)
$$

as $n \rightarrow \infty$ and

$$
\sup _{U_{1, n} \leq u, v \leq U_{k, n}}\left|\frac{s(u)-s(v)}{s(k / n)}\right|=O_{p}\left(\max \left(g_{1, n, 0}, g_{2, n, 0} \log k\right)\right) .
$$

Since $n k^{-1} U_{k+1, n} \rightarrow 1$ a.s. as $n \rightarrow \infty$, we may find for any $\varepsilon>0$ and for any $\lambda>1$, an integer $N_{0}$ such that for any $n \geq N_{0}$,

$$
\mathbb{P}\left(g_{1, n, 0} \leq g_{1, n}(p, \lambda), g_{2, n, 0} \leq g_{2, n}(b, \lambda)\right) \geq 1-\varepsilon .
$$

Hence $(C 3)$ implies

$$
\frac{S_{n}(1)}{\sigma_{n}(f) s(k / n)} \leq d_{n}(p, b, \lambda)\left(\sigma_{n}(f)\right)^{-1} \sum_{j=1}^{k} f(j) \rightarrow_{P} 0,
$$

where $d_{n}(p, b, \lambda)=\max \left(g_{1, n}, g_{2, n} \log k\right)$. Next

$$
\begin{aligned}
\frac{S_{n}(2)}{\sigma_{n}(f) s(k / n)} & =\sigma_{n}(f)^{-1} \sum_{j=1}^{k} f(j) \int_{U_{j, n}}^{U_{j+1, n}}\{s(t) / s(k / n)\} / t d t \\
& =\sigma_{n}(f)^{-1} \sum_{j=1}^{k} f(j) \int_{U_{j, n}}^{U_{j+1, n}} t^{-1} d t+\sigma_{n}(f)^{-1} \sum_{j=1}^{k} f(j) \int_{U_{j, n}}^{U_{j+1, n}}\{s(t) / s(k / n)-1\} / t d t \\
& \equiv S_{n}(2,1)+S_{n}(2,2) .
\end{aligned}
$$

We have, by (3.2) and the Malmquist representation (2.12),

$$
\begin{gathered}
\left|S_{n}(2,2)\right| \leq O_{p}(1) d_{n}(p, b, \lambda) \times \sigma_{n}(f)^{-1} \sum_{j=1}^{k} f(j) j^{-1} E_{j} \leq O_{p}(1) \times \\
\left\{d_{n}(p, b, \lambda) \times \sigma_{n}(f)^{-1} \sum_{j=1}^{k} f(j) j^{-1}\left(E_{j}-1\right)+d_{n}(p, b, \lambda) \times \sigma_{n}(f)^{-1} \sum_{j=1}^{k} f(j) j^{-1}\right\} .
\end{gathered}
$$

The first term tends to zero since $\sigma_{n}(f)^{-1} \sum_{j=1}^{k} f(j) j^{-1}\left(E_{j}-1\right)$ converges in distribution to a finite random variable by Lemma 1 in Section 6 and $d_{n}(p, b, \lambda) \rightarrow 0$ by $(C 0)$. The second also tends to zero by $(C 3)$. Finally, by the Malmquist representation (2.12), one arrives to

$$
S_{n}(2,1)=\sigma_{n}(f)^{-1} \sum_{j=1}^{k} f(j) j^{-1} E_{j}
$$

And this leads to

$$
S_{n}(2,1)-\left\{a_{n}(f) / \sigma_{n}(f)\right\}=\sigma_{n}(\tau)^{-1} \sum_{j=1}^{k} j^{\tau-1}\left(E_{j}-1\right),
$$

which converges in distribution to a $\mathcal{N}(0,1)$ random variable under $(\mathrm{K} 2)$ and to $\mathcal{L}(f)$ under $(\mathrm{K} 1)$ by Lemma 1 in Section 6. By sammerizing all these facts, we have proved that

$$
\left(\sigma_{n}(f) s(k / n)\right)^{-1}\left(T_{n}(f)-a_{n}(f) s(k / n)\right)
$$

converges in distribution to a $\mathcal{N}(0,1)$ random variable under $(\mathrm{K} 2)$ and to $\mathcal{L}(f)$ under $(\mathrm{K} 1)$.

Now let $F \in D\left(\phi_{1 / \gamma}\right)$, we have by (2.7) and the usual representations,

$$
\begin{aligned}
T_{n}(f) & =\sum_{j=1}^{k} f(j)\left\{\log \left(1+p\left(U_{j+1, n}\right)\right)-\log \left(1+p\left(U_{j, n}\right)\right)\right\}+\gamma \sum_{j=1}^{k} f(j) \log \left(U_{j+1, n} / U_{j, n}\right)+\sum_{j=1}^{k} f(j) \int_{U_{j, n}}^{U_{j+1, n}} b(t) / t d t \\
& \equiv S_{n}(1)+S_{n}(2)+S_{n}(3) .
\end{aligned}
$$


We have, for large values of $k$,

$$
\left|S_{n}(1) / \sigma_{n}(f)\right| \leq 2 g_{1, n, 0}(f)\left(\sigma_{n}(f)\right)^{-1} \sum_{j=1}^{k} f(j),
$$

where $g_{1, n, 0}$ is defined in (3.1), which tends to zero in probability by $(C 1)$ and (3.2). Next

$$
\begin{aligned}
\left|S_{n}(3) / \sigma_{n}(\tau)\right| & \leq g_{2, n, 0}(b)\left(\sigma_{n}(f)\right)^{-1} \sum_{j=1}^{k} f(j) \log \left(U_{j+1, n} / U_{j, n}\right) \\
& =g_{2, n, 0}(b) \sigma_{n}(f)^{-1} \sum_{j=1}^{k} f(j) j^{-1}\left(E_{j}-1\right)+g_{2, n, 0}(b) \sigma_{n}(f)^{-1} \sum_{j=1}^{k} f(j) j^{-1},
\end{aligned}
$$

where $g_{2, n, 0}$ defined in (3.1). Then $S_{n}(3) / \sigma_{n}(f) \rightarrow 0$ by $(C 3)$ and Lemma 1 and the methods described above. Finally, always by Lemma 1 ,

$$
\left\{\left(S_{n}(3)-\gamma a_{n}(f)\right\} / \sigma_{n}(f)=\gamma \sigma_{n}(f)^{-1} \sum_{j=1}^{k} f(j) j^{-1}\left(E_{j}-1\right)=\gamma V_{n} *(f) .\right.
$$

We recall that $V_{n}(f)$ and $V_{n} *(f)$ are both defined in Lemma 1 and its proofs in Equations (6.1 - 6.4). We conclude the proof by noticing that the right member this latter equation converges in distribution to a $\mathcal{N}\left(0, \gamma^{2}\right)$ random variable under $(\mathrm{K} 2)$ and to $\gamma L(f)$ under $(\mathrm{K} 1)$, by Lemma 1.

From these proofs, we get two intermediate results towards the functional laws. The first concerns the asymptotic law in finite distributions.

Corollary 1 Suppose that the hypotheses $(C 0),(C 1),(C 2)$ and $(C 3)$ hold and for any $\left(f_{1}, f_{2}\right) \in \mathcal{F}_{1}^{2} \cup \mathcal{F}_{2}^{2}$,

$$
\lim _{n \rightarrow \infty} \frac{1}{\sigma_{n}\left(f_{1}\right) \sigma_{n}\left(f_{2}\right)} \sum_{j=1}^{k} f_{1}(j) f_{2}(j) j^{-2}=\Gamma\left(f_{1}, f_{2}\right) \text { exists. }
$$

Then the finite-distributions of $\left\{\left(s(k / n) \sigma_{n}(f)\right)^{-1}\left(T_{n}(f)-a_{n}(f) s(k / n)\right), f \in \mathcal{F}_{1}\right\}$ weakly converge to those of the process $\mathcal{L}$, for $F \in D(\Lambda)$ and the finite-distributions of $\left\{\sigma_{n}(f)^{-1}\left(T_{n}(f)-a_{n}(f), f \in \mathcal{F}_{1}\right\}\right.$ weakly converge to those of the process $\gamma \mathcal{L}$, for $F \in D\left(\varphi_{1 / \gamma}\right)$.

And the finite-distributions of $\left\{\left(s(k / n) \sigma_{n}(f)\right)^{-1}\left(T_{n}(f)-a_{n}(f) s(k / n)\right), f \in \mathcal{F}_{2}\right\}$ weakly converge to those of a Gaussian process $\mathbb{G}$ of covariance function

$$
\Gamma\left(f_{1}, f_{2}\right)=\lim _{n \rightarrow \infty} \frac{1}{\sigma_{n}\left(f_{1}\right) \sigma_{n}\left(f_{2}\right)} \sum_{j=1}^{k} f_{1}(j) f_{2}(j) j^{-2}
$$

and, for $F \in D(\Lambda)$, the finite-distributions of $\left\{\sigma_{n}(f)^{-1}\left(T_{n}(f)-a_{n}(f), f \in \mathcal{F}_{2}\right\}\right.$ weakly converge to those of $\gamma \mathbb{G}$, for $F \in D\left(\varphi_{1 / \gamma}\right)$.

Proof. Put $V_{n}(0, f)=\left(s(k / n) \sigma_{n}(f)\right)^{-1}\left(T_{n}(f)-a_{n}(f) s(k / n)\right)$ and $V_{n}(1, f)=\sigma_{n}(f)^{-1}\left(T_{n}(f)-a_{n}(f)\right)$ for a fixed $f \in \mathcal{F}_{h}, h=1,2$. For a finite family $\left(f_{1}, f_{2}, \ldots, f_{s}\right) \in f \in \mathcal{F}_{h}^{s}, h=1,2,0<s \in \mathbb{N}^{*}$, we have by the proof of Theorem 1 , for each $1 \leq i \leq s$,

$$
V_{n}\left(0, f_{i}\right)=V_{n}^{*}\left(f_{i}\right)+o_{P}(1)
$$

for $F \in D(\Lambda)$ and

$$
V_{n}\left(1, f_{i}\right)=\gamma V_{n}^{*}\left(f_{i}\right)+o_{P}(1)
$$

for $F \in D\left(\varphi_{1 / \gamma}\right)$. From now, we conclude by applying Lemma 2 which establishes the asymptotic laws of $\left(V_{n}^{*}\left(f_{1}\right)\right.$, $\left.\ldots, V_{n}^{*}\left(f_{s}\right)\right)$ under the assumptions of the Lemma.

This corollary makes a good transition towards the functional law. In order to state a further step, we need the following uniform conditions on the distribution function $F$. Define for some $\lambda>1$,

$$
\sup _{f \in \mathcal{F}_{h}} d_{n}(p, b, \lambda) \rightarrow 0
$$




$$
\begin{gathered}
\sup _{f \in \mathcal{F}_{h}} g_{1, n}(f, \lambda)\left(\sigma_{n}(f)\right)^{-1} \sum_{j=1}^{k} f(j) \rightarrow 0, \\
\sup _{f \in \mathcal{F}_{h}} g_{2, n}(b, \lambda)\left(\sigma_{n}(f)\right)^{-1} \sum_{j=1}^{k} f(j)
\end{gathered}
$$

and

$$
\sup _{f \in \mathcal{F}_{h}} d_{n}(p, b, \lambda) \sigma_{n}(f)^{-1} \sum_{j=1}^{k} f(j) \rightarrow 0
$$

These conditions are set so that the $o_{P}(1)$ in (3.5) and (3.6) hold uniformly in our classes. We thus begin to state this:

Corollary 2 Assume that the uniform conditions (KU1), (KU2a), (KU2b), (CU0) (CU1), (CU2) and (CU3) hold - when appropriate - in Theorem 2. Finally let $\mathcal{F}_{a}$ be a nonvoid family of functions satisfying (KU1) or, a nonvoid family of functions satisfying $(K U 2 a-b)$. Suppose that $\left\{V_{n}^{*}(f), f \in \mathcal{F}_{a}\right\}$ weakly converges in $\ell^{\infty}\left(\mathcal{F}_{a}\right)$. Then, uniformly in $f \in \mathcal{F}_{a}$,

$$
\left(s(k / n) \sigma_{n}(f)\right)^{-1}\left(T_{n}(f)-a_{n}(f) s(k / n)\right)=V_{n}^{*}(f)+o_{P}^{*}(1)
$$

for $F \in D(\Lambda)$ and

$$
\left(a_{n}(f) / \sigma_{n}(f)\right)\left(T_{n}(f) / a_{n}-\gamma\right)=\gamma V_{n}^{*}(f)+o_{P}^{*}(1)
$$

for $F \in D\left(\phi_{1 / \gamma}\right)$.

Proof. Put

$$
V_{n}(0, f)=\left(s(k / n) \sigma_{n}(f)\right)^{-1}\left(T_{n}(f)-a_{n}(f) s(k / n)\right)
$$

and

$$
V_{n}(1, f)=\left(a_{n}(f) / \sigma_{n}(f)\right)\left(T_{n}(f) / a_{n}-\gamma\right) .
$$

When the uniformity hypotheses (KU1) or,(KU2a) and (KU2b) hold, we surely have

$$
V_{n}(0, f)=V_{n}^{*}(f)\left(1+o_{P}^{*}(1)\right)+o_{P}^{*}(1) \text { and } V_{n}(1, f)=\gamma V_{n}^{*}(f)\left(1+o_{P}^{*}(1)\right)+o_{P}^{*}(1),
$$

uniformly in $f \in \mathcal{F}_{h}$. Now let $\mathcal{F}_{a}$ a subset of $\mathcal{F}_{1}$ such that $\left\{V_{n}^{*}(f), f \in \mathcal{F}_{a}\right\}$ weakly converges, say to $\mathbb{G}$ in $\ell^{\infty}\left(\mathcal{F}_{a}\right)$. Then $\|\mathbb{G}\|_{\mathcal{F}_{a}}^{*}<\infty$. Since, by the continuity theorem, $\left\|V_{n}^{*}\right\|_{\mathcal{F}_{a}} \sim\|\mathbb{G}\|_{\mathcal{F}_{a}}^{*}$, we get

$$
V_{n}(0, f)=V_{n}^{*}(f)+o_{P}^{*}(1)
$$

uniformly in $f \in \mathcal{F}_{a}$ for $F \in D(\Lambda)$. The other cases are proved similarly.

\section{The Functional Law of $T_{n}(f)$}

We shall use here (3.7). Recall and denote

$$
V_{n}^{*}(f)=\sigma_{n}(f)^{-1}\left(V_{n}(f)-a_{n}(f)\right)=\sum_{j=1}^{k} \frac{f(j) j^{-1}}{\sigma_{n}(f)}\left(E_{j}-1\right)=: \sum_{j=1}^{k} Z_{j, n}(f) .
$$

Then, our main tools for handling these stochastic processes are Theorem 2.11.1 and Theorem 2.11.9 of (van der Vaart \& Wellner, 1996) on uniform laws of sums of independent stochastic processes. We will then need the basic frame of these theorems.

Next, we shall possibly consider sub-families $\mathcal{F}$ of of $\mathcal{F}_{h}(h=1,2)$ formed by functions $f: \mathbb{N} \backslash 0 \longmapsto \mathbb{R}_{+} \backslash 0$ satisfying this measurability assumption, that is for each $h=1,2$, for each $\delta>0$ and for each $\left(e_{1}, \ldots, e_{n}\right) \in$ $\{-1,0,1\}^{n}$, for each $p=1,2$,

$$
\sup _{\left(f_{1}, f_{2}\right) \in \mathcal{F}_{h}, \rho\left(f_{1}, f_{2}\right) \leq \delta} \sum_{j=1}^{k} e_{j}\left|\sigma_{n}^{-1}\left(f_{1}\right) f_{1}(j) j^{-1}-\sigma_{n}^{-1}\left(f_{2}\right) f_{2}(j) j^{-1}\right|^{p} E_{j}^{p},
$$


is measurable, where $\rho$ is a semimetric space on $\mathcal{F}$. Precisely, we introduce the two classes. Let subfamilies $\mathcal{F}_{h, 0}$ of $\mathcal{F}_{h}(h=1,2)$ such that each of them is equipped with a semimetric $\rho_{h}$ such that $\left(F_{h, 0}, \rho_{h}\right)$ is totally bounded, and that the measurability of (MES) holds. We may also need the random semimetric

$$
d_{n}^{2}\left(f_{1}, f_{2}\right)=\sum_{j=1}^{k}\left(\sigma_{n}^{-1}\left(f_{1}\right) f_{1}(j) j^{-1}-\sigma_{n}^{-1}\left(f_{2}\right) f_{2}(j) j^{-1}\right)^{2}\left(E_{j}-1\right)^{2} .
$$

Now suppose that $\left(\mathcal{F}_{h, 0}, \|||\right)$ be a normed space verifying the Riesz property. Let us define, as in van der Vaart and Wellner (1996, p. 211), the bracketing number $N_{[]}\left(\varepsilon, \mathcal{F}_{h, 0}, L_{2}^{n}\right)$ as the minimal number of sets $N_{\varepsilon}$ in a partition $\mathcal{F}_{h, 0}=\bigcup_{j=1}^{N_{\varepsilon}} \mathcal{F}_{\varepsilon j}^{n}$ of the index set into sets $\mathcal{F}_{\varepsilon j}^{n}$ such that, for every partitioning set $\mathcal{F}_{\varepsilon j}^{n}$,

$$
\sum_{i=1}^{k} \mathbb{E}^{*} \sup _{f, g \in F_{\varepsilon j}^{n}}\left|Z_{n i}\left(f_{1}\right)-Z_{n i}\left(f_{2}\right)\right|^{2} \leq \varepsilon^{2}
$$

We have our first version of the functional laws of $T_{n}(f)$.

Theorem 3 Suppose that for each $h=1,2$, we have

$$
\sup _{\left(f_{1}, f_{2}\right) \in \mathcal{F}_{h, 0}^{2}, \rho_{h}\left(f_{1}, f_{2}\right) \leq \delta_{n}} \sum_{j=1}^{k(n)}\left(f_{1}(j) /\left(j \sigma_{n}(f)\right)-f_{2}(j) /\left(j \sigma_{n}(f)\right)\right)^{2} \rightarrow 0
$$

as $\delta_{n} \downarrow 0$ as $n \uparrow+\infty$, and

$$
\int_{0}^{\delta_{n}} \sqrt{\log N_{[]}\left(\epsilon, \mathcal{F}_{h}, d_{n}\right)} d \epsilon \rightarrow 0,
$$

as $n \uparrow \infty$, where $N_{[]}\left(\epsilon, \mathcal{F}_{h, 0}, d_{n}\right)$ is the $\epsilon$-entropy number of $\mathcal{F}_{h, 0}$ with respect to the semi-metric $d_{n}$, that is the minimal number of $d_{n}$-balls of radius at most $\epsilon$ needed to cover $\mathcal{F}_{h}$.

Let $F \in D(\Lambda)$ and suppose that (CUO) and (CU3) hold. Then

$$
\left\{\left(s(k / n) \sigma_{n}(f)\right)^{-1}\left(T_{n}(f)-s(k / n) a_{n}(f)\right), f \in \mathcal{F}_{1}\right\}
$$

converges to a Gaussian process in $\ell^{\infty}\left(\mathcal{F}_{2,0}\right)$ with covariance function

$$
\Gamma\left(f_{1}, f_{2}\right)=\lim _{n \rightarrow \infty} \sum_{j=1}^{k(n)}\left\{\sigma_{n}\left(f_{1}\right) \sigma_{n}\left(f_{2}\right)\right\}^{-1} f_{1}(j) f_{2}(j) j^{-2} \leq 1 .
$$

And $\left\{\left(s(k / n) \sigma_{n}(f)\right)^{-1}\left(T_{n}(f)-s(k / n) a_{n}(f)\right), f \in \mathcal{F}_{1,0}\right\}$ converges to a stochastic process $\left\{\mathcal{L}(f), f \in \mathcal{F}_{1,0}\right\}$ in $\ell^{\infty}\left(\mathcal{F}_{1,0}\right)$ with covariance function $\Gamma\left(f_{1}, f_{2}\right)$. The finite distributions of $\left(\mathcal{L}\left(f_{1}\right), \ldots, \mathcal{L}\left(f_{S}\right)\right)$ are characterized by the generating moments function

$$
\left(t_{1}, \ldots, t_{S}\right) \mapsto \prod_{j=1}^{+\infty} \exp \left(\sum_{s=1}^{S} t_{s} f_{s}(j) j^{-1}\right)\left(1-\sum_{s=1}^{S} t_{s} f_{s}(j) j^{-1}\right)
$$

for $\left|t_{s}\right| \leq 1 / S, s=1, \ldots, S$.

Let $F \in D\left(\phi_{1 / \gamma}\right)$ and suppose that (CU1) and (CU2) hold. Then

$$
\left\{\left(a_{n}(f) / \sigma_{n}(f)\right)\left(T_{n}(f) / a_{n}-\gamma\right), f \in \mathcal{F}_{2,0}\right\}
$$

converges to a Gaussian process in $\ell^{\infty}\left(\mathcal{F}_{2,0}\right)$ with covariance function $\Gamma$.

And $\left\{\left(a_{n}(f) / \sigma_{n}(f)\right)\left(T_{n}(f) / a_{n}-\gamma\right) / \gamma, f \in \mathcal{F}_{1,0}\right\}$ converges to a stochastic process in $\ell^{\infty}\left(\mathcal{F}_{1,0}\right)$ with covariance function $\gamma^{2} \Gamma$ and finite distribution characterized by (4.1).

Proof. We begin by applying Corollary 2. For $T_{n}^{*}(f)=\left(s(k / n) \sigma_{n}(f)\right)^{-1}\left(T_{n}(f)-a_{n}(f) s(k / n)\right)$ for $F \in D(\Lambda)$ or $T_{n}^{*}(f)=\left(a_{n}(f) / \sigma_{n}(f)\right)\left(T_{n}(f) / a_{n}-\gamma\right)$ for $F \in D\left(G_{1 / \gamma}\right)$, we have

$$
T_{n}^{*}(f)=V_{n}^{*}(f)+o_{P}^{*}(1)
$$


uniformly in $f \in \mathcal{F}_{h}$ and hence uniformly in $f \in \mathcal{F}_{h, 0}(h=1,2)$. From there, we apply Theorem 2.11.1 of (van der Vaart \& Wellner, 1996) on the uniform behavior of the stochastic processes

$$
V_{n}^{*}(f)=\sigma_{n}(f)^{-1}\left(V_{n}(f)-a_{n}(f)\right)=\sum_{j=1}^{k} \frac{f(j) j^{-1}}{\sigma_{n}(f)}=: \sum_{j=1}^{k} Z_{j, n}(f),
$$

indexed by $f \in \mathcal{F}_{h, 0}$. All the assumptions of Theorem 2.11.1 of (van der Vaart \& Wellner, 1996) have already been taken into account in our statement, except this one,

$$
\mathbb{E}^{*}\left\{\left\|Z_{j, n}\right\|_{\mathcal{F}_{h}}^{2} I_{\left(\left\|Z_{j, n}\right\|_{\mathcal{F}_{h}}>\eta\right)}\right) \rightarrow 0 \text {, as } n \rightarrow \infty,
$$

for any $\eta>0$, and the convergence of the covariance function. But the $\left\|Z_{j, n}\right\|$ are measurable and

$$
\mathbb{E}\left\|Z_{j, n}\right\|_{\mathcal{F}_{1}}=\max \{f(j) / j, j \geq 1\} / \sigma_{n}(f) \rightarrow 0,
$$

because of $(K U 1)$ and

$$
\mathbb{E}\left\|Z_{j, n}\right\|_{\mathcal{F}_{2}} \leq B_{n} \rightarrow 0
$$

because of $(K U 2 a)$. This proves ( $L 3)$. As to the covariance functions which are

$$
\Gamma_{n}\left(f_{1}, f_{2}\right)=\sum_{j=1}^{k(n)}\left\{\sigma_{n}\left(f_{1}\right) \sigma_{n}\left(f_{2}\right)\right\}^{-1} f_{1}(j) f_{2}(j) j^{-2},
$$

we notice by the Cauchy-Schwartz inequality that they are bounded by the unity. For $h=1$, we have

$$
\Gamma_{n}\left(f_{1}, f_{2}\right)=\frac{1}{\sqrt{A\left(2, f_{1}\right) A\left(2, f_{2}\right)}} \sum_{j=1}^{\infty} f_{1}(j) f_{2}(j) j^{-2} \leq 1,
$$

while for $h=2$, the condition $(K U 2 a)$ guarantees the desired result. We thus conclude that $\left\{T_{n}^{*}(f), f \in \mathcal{F}_{h, 0}\right\}$ weakly converges in $\ell^{\infty}\left(\mathcal{F}_{h, 0}\right)$ for each $h=1,2$. Now, by Theorem 2 and Corollary 1 , we know that the weak limit is either $\mathcal{L}$ defined by (4.1) or a Gaussian process $\mathbb{G}$ of covariance function $\Gamma$.

Now, we present the second version which is more general since we do not require the measurability assumption so that we consider the whole spaces $\mathcal{F}_{h}(h=1,2)$.

Theorem 4 If $\left(\mathcal{F}_{h},||||\right)$ is a normed space verifying the Riesz property, then the results of Theorem 3 hold when

$$
\int_{0}^{\delta_{n}} \sqrt{\log N_{[]}\left(\epsilon, \mathcal{F}_{h}, L_{2}^{n}\right)} d \epsilon \rightarrow 0,
$$

as $n \rightarrow \infty$, in place of $(L 2)$, provided that the $Z_{j, n}$ have finite second moments.

Proof. It is achieved by applying Theorem 2.11 .9 of van der Vaart and Wellner (1996) in the proof of Theorem 3, that requires $(L 1),(L 2),(L 4)$ and that the $Z_{j, n}$ have finite second moments. But $(L 2)$ and the last condition hold. Thus $(L 1)$ and $(L 4)$ together ensure the results of the theorem.

\section{Special Classes}

We specialize these results for the special class of the monotone functions $f_{\tau}(j)=j^{\tau}, 0<\tau$. We will show here, in this example, how to derive particular laws for special classes from our general results. We know from Dème, Lo and Diop (2010) and Diop and Lo (2009) that $T_{n}\left(f_{\tau}\right)$ is asymptotically normal for $\tau>1 / 2$ while it asymptotically follows a $\mathcal{L}\left(f_{\tau}\right)$ type-law for $0<\tau<1 / 2$, under usual conditions of the $d . f$. $G$. We handle here the uniform asymptotic behavior for these two range values of $\tau$ : $[0,1 / 2]$ and $[1 / 2,+\infty)$. For the first case, we apply Theorem 3 and for the second, Theorem 4. First, let $0<a<b<1 / 2$ and put,

$$
\mathcal{F}_{0}(a, b)=\left\{f(j)=j^{\tau}, a \leq \tau \leq b\right\} .
$$

We have

Corollary 3 Let $0<a<b<1 / 2$ and $\mathcal{F}_{0}=\mathcal{F}_{0}(a, b)=\left\{f(j)=j^{\tau}, 0<a \leq \tau \leq b<1 / 2\right\}$. Then, 
(1) if $F \in D\left(G_{0}\right)$ and if (CU2) and (CU2) hold. Then

$$
\left\{\left(s(k / n) \sigma_{n}(f)\right)^{-1}\left(T_{n}(f)-s(k / n) a_{n}(f)\right), f \in \mathcal{F}_{0}\right\}
$$

weakly converges to $\left\{\mathcal{L}(f), f \in \mathcal{F}_{0}\right\}$.

(2) if $F \in D\left(G_{1 / \gamma}\right), \gamma>0$, and if (CU1) holds. Then

$$
\left\{\left(a_{n} / \sigma_{n}(f)\right)\left(T_{n}(f) / a_{n}(f)-\gamma\right), f \in \mathcal{F}_{0}\right\}
$$

weakly converges to $\left\{\gamma \mathcal{L}(f), f \in \mathcal{F}_{0}\right\}$.

Proof. We apply here Theorem 3. We have

$$
\mathcal{F}_{0,1}=\left\{g(j)=j^{-(b-\tau)}, a \leq \tau \leq b\right\}
$$

For $f(j)=j^{\tau}$, denote $g_{f}(j)=j^{-(b-\tau)}$, that is

$$
f(j) / j=g_{f}(j) \times j^{-(1-b)} .
$$

In this case, put

$$
V_{n}^{* *}(f)=\left(V_{n}(f)-a_{n}(f)\right)=\sum_{j=1}^{k} g_{f}(j)\left(E_{j}-1\right) j^{-(1-b)}=: \sum_{j=1}^{k} Z_{j, n}(f)
$$

We have

$$
\begin{aligned}
\rho_{n}^{2}\left(f_{1}, f_{2}\right)=\mathbb{E} \sum_{j=1}^{k}\left(Z_{j, n}\left(f_{1}\right)-Z_{j, n}\left(f_{2}\right)\right)^{2}=\sum_{j=1}^{k}\left(f_{1}(j)-f_{2}(j)\right)^{2} j^{-2} \\
\leq \rho^{2}\left(f_{1}, f_{2}\right)=\sum_{j=1}^{\infty}\left(f_{1}(j)-f_{2}(j)\right)^{2} j^{-2}=\sum_{j=1}^{\infty}\left(g_{f_{1}}(j)-g_{f_{2}}(j)\right)^{2} j^{-2(1-b)} .
\end{aligned}
$$

We point out that $\rho^{2}\left(f_{1}, f_{2}\right)$ is nothing else but $\left\|g_{f_{1}}-g_{f_{2}}\right\|_{L_{2}\left(\mathcal{F}_{0,1}, \mathbb{Q}\right)}^{2}$ for the probability measure on $\mathbb{N}$,

$$
\mathbb{Q}=A(2, b)^{-1} \sum_{j=1}^{\infty} j^{-2(1-b)} \delta_{j}
$$

for

$$
A(2, b)=\sum_{j=1}^{\infty} j^{-2(1-b)}<\infty
$$

For such monotone functions $g_{f}: \mathbb{N} \mapsto[0,1]$, we have by virtue of Theorem 2.7.5 of van der Vaart and Wellner (1996) that for some $K>0$, any $\epsilon>0$,

$$
N_{[]}\left(\epsilon, \mathcal{F}_{0,1}, L_{2}(\mathbb{Q})\right) \leq \exp \left(K \epsilon^{-1}\right)
$$

This means that $\left(\mathcal{F}_{0}, \rho\right)$ is totally bounded and $(L 1)$ is reduced to

$$
\sup _{\rho\left(f_{1}, f_{2}\right) \leq \delta_{n}} \rho_{n}\left(f_{1}, f_{2}\right) \leq \delta_{n} \rightarrow 0,
$$

which is trivial. In the same spirit

$$
A(2, b, \omega)=\sum_{j=1}^{\infty} j^{-2(1-b)}\left(E_{j}-1\right)^{2}(\omega)
$$

is almost surely finite and

$$
Q_{0}(\omega)=A(2, b, \omega)^{-1} \sum_{j=1}^{\infty} j^{-2(1-b)}\left(E_{j}-1\right)^{2}(\omega) \delta_{j}
$$


is a probability measure for almost all $\omega$. And we have

$$
0 \leq d_{n}^{2}\left(f_{1}, f_{2}\right) \rightarrow d^{2}\left(f_{1}, f_{2}\right)=\left\|g_{f_{1}}-g_{f_{2}}\right\|_{L_{2}\left(\mathcal{F}_{0,1}, \mathbb{Q}_{0}\right)} .
$$

This convergence is a continuous one since $\tau$ lies on the compact set $[a, b]$ (see Subsection 7.2. in the Appendix 7 for such results). Thus, uniformly in $\tau \in[a, b]$, for large values of $n$,

$$
0 \leq d_{n}^{2}\left(f_{1}, f_{2}\right) \geq 0.25 d^{2}\left(f_{1}, f_{2}\right) .
$$

We may use the same results of (van der Vaart, \& Wellner, 1996) to get for some $K>0$ and for any $\epsilon>0$,

$$
\log N_{[]}\left(\epsilon, \mathcal{F}_{0}, d_{n}\right) \leq \log N_{[]}\left(\epsilon, \mathcal{F}_{0}, d / 2\right) \leq K(\epsilon / 2)^{-1} .
$$

This ensures (L2). The covariance function is, for $f_{1}(j)=j^{\tau_{1}}$ and $f_{2}(j)=j^{\tau_{2}}$,

$$
\Gamma^{*}\left(f_{1}, f_{2}\right)=\lim _{n \rightarrow \infty} \sum_{j=1}^{k} f_{1}(j) f_{2}(j) j^{-2}=\sum_{j=1}^{\infty} j^{-\left(2-\tau_{1}-\tau_{2}\right)} \leq \sum_{j=1}^{\infty} j^{-2(1-b)}=A(2, b) .
$$

As to the measurability hypothesis, it is readily seen that the following supremum

$$
\sup _{\left(f_{1}, f_{2}\right) \in \mathcal{F}_{h}, \rho\left(f_{1}, f_{2}\right) \leq \delta_{n}} \sum_{j=1}^{k} e_{j}\left|\sigma_{n}^{-1}\left(f_{1}\right) f_{1}(j) j^{-1}-\sigma_{n}^{-1}\left(f_{2}\right) f_{2}(j) j^{-1}\right|^{p} E_{j}^{p}
$$

is achieved through the rational values of $\tau$ in $[a, b]$, and then, is measurable. This achieves the proof.

Corollary 4 Let $1 / 2<a<b>1$ and $\mathcal{F}_{1}(a, b)=\left\{f(j)=j^{\tau}, 0<a \leq \tau \leq b\right\}$.

(1) If $F \in D\left(G_{0}\right)$ and if (CU3). Then

$$
\left\{\left(s(k / n) \sigma_{n}(f)\right)^{-1}\left(T_{n}(f)-s(k / n) a_{n}(f)\right), f \in \mathcal{F}_{0}\right\}
$$

weakly converges to a Gaussian process $\mathbb{G}$ of covariance function $\Gamma$.

(2) If $F \in D\left(G_{1 / \gamma}\right), \gamma>0$, and if (CU1) holds. Then

$$
\left\{\left(a_{n} / \sigma_{n}(f)\right)\left(T_{n}(f) / a_{n}(f)-\gamma\right), f \in \mathcal{F}_{0}\right\}
$$

weakly converges to the Gaussian process $\gamma \mathbb{G}$.

Proof. We apply here Theorem 4. But, we begin by returning to the simple scheme, that is, to fixed $f \in \mathcal{F}_{1}(a, b)$. Let $f(j)=j^{\tau}$. We have

$$
B(n, f)=O\left((\log k)^{-1}\right),
$$

$\tau=1 / 2$,

$$
B(n, f)=O\left(k^{-(2 \tau-1)}\right)
$$

for $0<\tau<1 / 2$ and

$$
B(n, f)=O\left(k^{-\tau}\right),
$$

for $\tau>1$. Then $(K 1)$ holds and this leads to the normal case in Theorem 2 for each $\tau \geq 1 / 2$. Next, for $f_{1}(j)=j^{\tau_{1}}$ and $f_{2}(j)=j^{\tau_{2}}, \tau_{1}>1 / 2, \tau_{2}>1 / 2$,

$$
\lim _{n \rightarrow \infty} \sum_{j=1}^{k(n)}\left\{\sigma_{n}\left(f_{1}\right) \sigma_{n}\left(f_{2}\right)\right\}^{-1} f_{1}(j) f_{2}(j) j^{-2}=\frac{\sqrt{\left(2 \tau_{1}-1\right)\left(2 \tau_{2}-1\right)}}{\tau_{1}+\tau_{2}-1}=\Gamma\left(f_{1}, f_{2}\right)<\infty .
$$

And for $\tau_{1}=1 / 2$ and $\tau_{2}>1 / 2$

$$
\sum_{j=1}^{k(n)}\left\{\sigma_{n}\left(f_{1}\right) \sigma_{n}\left(f_{1}\right)\right\}^{-1} f_{1}(j) f_{2}(j) j^{-2} \sim \sqrt{2 \tau_{2}-1}(\log k) k^{\left(2 \tau_{2}-1\right) / 2},
$$


that is, $\mathcal{F}_{1}(a, b)$ satisfies $(K U 2 a)$. This also implies that we have the finite-distributions weak normality for $\tau>1 / 2$. It also fulfills $(K U 2 b)$ since

$$
B(n)=\sup _{f \in \mathcal{F}_{1}(a, b)} B(n, f) \leq k^{-b} \rightarrow 0
$$

Consider here

$$
V_{n}^{* *}(f)=\sigma_{n}(f)^{-1}\left(V_{n}(f)-a_{n}(f)\right)=\sum_{j=1}^{k} f(j) /\left(j \sigma_{n}(f)\right)\left(E_{j}-1\right)=: \sum_{j=1}^{k} Z_{j, n}(f)
$$

We have

$$
\begin{gathered}
\rho_{n}^{2}\left(f_{1}, f_{2}\right)=\mathbb{E} \sum_{j=1}^{k}\left(Z_{j, n}\left(f_{1}\right)-Z_{j, n}\left(f_{2}\right)\right)^{2}=2-\frac{2}{\sigma_{n}(f 1) \sigma_{n}\left(f_{2}\right)} \sum_{j=1}^{k} f_{1}(j) f_{2}(j) j^{-2} \\
\rightarrow 2\left(1-\Gamma\left(f_{1}, f_{2}\right)\right)=\sum_{j=1}^{\infty}\left\{\left(f_{1}(j) /\left(j \sigma_{n}\left(f_{1}\right)\right)-\left(f_{2}(j) /\left(j \sigma_{n}\left(f_{2}\right)\right)\right\}^{2} .\right.\right.
\end{gathered}
$$

By routine calculations and by a continuity argument based on the remark that our $\tau^{\prime} s$ are in the compact set $[a, b]$, we may show that

$$
\rho_{n}^{2}\left(f_{1}, f_{2}\right)<3\left(1-\Gamma\left(f_{1}, f_{2}\right)\right)
$$

for large values of $n$. Since we use this type of arguments many times, we show in Subsection 7.2 of the appendix 7 the exact proof of (5.2). But

$$
1-\Gamma\left(f_{1}, f_{2}\right)=\left(1-\frac{\sqrt{\left(2 \tau_{1}-1\right)\left(2 \tau_{2}-1\right)}}{\tau_{1}+\tau_{2}-1}\right)
$$

For $\tau_{1}-\tau_{2}=\delta>0$

$$
1-\Gamma\left(f_{1}, f_{2}\right)=\frac{\left(2 \tau_{2}-1\right)+\delta-\sqrt{\left(2 \tau_{2}-1\right)^{2}+2 \delta\left(2 \tau_{2}-1\right)}}{\tau_{1}+\tau_{2}-1} .
$$

We use a Taylor expansion of a first order, to get for $1 / 2<a<\tau_{1}, \tau_{2}<b, 3\left(1-\Gamma\left(f_{1}, f_{2}\right)\right) \leq B(a, b) \delta$, where $B(a, b)$ depends only on $a$ and $b$. Thus for a fixed $\delta$, for large values of $k$,

$$
\rho_{n}^{2}\left(f_{1}, f_{2}\right) \leq B(a, b) \delta
$$

We may now take the metric $\rho\left(f_{1}, f_{2}\right)=\left\|\tau_{1}-\tau_{2}\right\|$, for which $\left(\mathcal{F}_{1}(a, b), \rho_{2}\right)$ is a Riesz space totally bounded and we surely obtain

$$
\sup _{\rho_{2}\left(f_{1}, f_{2}\right) \leq \delta_{n}} \rho_{n}\left(f_{1}, f_{2}\right) \leq 4 B(a, b) \delta_{n} \rightarrow 0 .
$$

This gives the $(L 1)$ hypothesis. Now, to conclude the proof by establishing the functional law as already described in Corollary 1 , we have to prove that $(L 4)$ holds for $\left(\mathcal{F}_{1}(a, b), \rho_{2}\right)$ with partitions not depending on $n$, so that ( $\left.L 2\right)$ is unnecessary. Since the proof concerning $(L 4)$ is very technical, we state it Subsection 7.1 of the Appendix 7.

\section{Technical Lemmas}

Define the following conditions

$$
\left.A(2, f)=\sum_{j=1}^{\infty} f(j)^{2} j^{-2} \in\right] 0,+\infty[
$$

with the notation (well-defined since $f>0$ )

$$
A(n, f)=\sum_{j=1}^{\infty} f(j)^{n} j^{-n}
$$

and

$$
\limsup _{n \rightarrow+\infty} \sigma_{n}(f)^{-1} \max \left\{f(j) j^{-1}, 1 \leq j \leq k\right\}=\limsup _{n \rightarrow+\infty} B(n, f)=0
$$

We begin by this simple lemma where we suppose that we are given a sequence of independent and uniformly distributed random variables $U_{1}, U_{2}, \ldots$ as in $(2.11)$. 
Lemma 1 Let

$$
V_{n}(f)=\sum_{j=1}^{k} f(j) \log \left(\frac{U_{j+1, n}}{U_{j, n}}\right)
$$

If (K2) holds,

$$
\sigma_{n}^{-1}(f)\left(V_{n}(f)-a_{n}(f)\right) \sim \mathcal{N}(0,1)
$$

and if (K1) holds,

$$
\sigma_{n}^{-1}(f)\left(V_{n}(f)-a_{n}(f)\right) \leadsto \mathcal{L}(f),
$$

where $a_{n}(f)$ and $\sigma_{n}(f)$ are defined in (1.2) and

$$
\mathcal{L}(\tau)=A(2, f) \sum_{j=1}^{\infty} f(j) j^{-1}\left(E_{j}-1\right),
$$

is a centered and reduced random variable with all finite moments.

Proof. By using the Malmquist representation (2.12), we have

$$
V_{n}(f)=\sum_{j=1}^{k} f(j) j^{-1} E_{j}
$$

It follows that $\mathbb{E}\left(V_{n}(f)\right)=a_{n}(f)$ and $\mathbb{V} \operatorname{ar}\left(V_{n}(f)\right)=\sigma_{n}^{2}(f)$. Put

$$
V_{n}^{*}(f)=\sigma_{n}(f)^{-1}\left(V_{n}(f)-a_{n}(f)\right)
$$

Then

$$
V_{n}^{*}(f)=\sigma_{n}(f)^{-1} \sum_{j=1}^{k} f(j) j^{-1}\left(E_{j}-1\right) .
$$

First suppose that (K1) holds, that is $\sigma_{n}(f) \rightarrow A(2, f)^{-1 / 2} \in[0,1]$. Then

$$
V_{n}^{*}(f) \rightarrow A(2, f)^{-1 / 2} \sum_{j=1}^{\infty} f(j) j^{-1}\left(E_{j}-1\right)=\mathcal{L}(f)
$$

Now, we have to prove that $\mathcal{L}(f)$ is a well-defined random variable with all finite moments. The moment characteristic function of $V_{n}^{*}(f)$ is

$$
\psi_{V_{n}^{*}(f)}(t)=\exp \left(-A(2, f)^{-1 / 2} \sum_{j=1}^{k} f(j) j^{-1}(i t)\right) \prod_{j=1}^{k}\left(1-i t \times f(j) j^{-1} A(f)^{-1 / 2}\right)^{-1} .
$$

By using the development of $\log (1-\cdot)$, and, by the Lebesgues Theorem, one readily proves that

$$
\psi_{V_{n}^{*}(\tau)}(t)=\exp \left(\sum_{j=1}^{k} \sum_{n=2}^{\infty} \frac{(i t)^{n}}{n} f(j)^{n} j^{-n} A(2, f)^{-n / 2}\right) \rightarrow \psi_{\infty}(t)=\exp \left(\sum_{n=2}^{\infty} \frac{(i t)^{n}}{n} A(n, f) A(2, f)^{-n / 2}\right) .
$$

We note that if $A(2, f)<\infty$, then $A(n, f)$ is also finite for any $n \geq 2$, since the general term (in $j)$ of $A(n, f)$ is less than that of $A(2, f)$, for large values of $j$. This concludes the proof when $(K 1)$ holds.

Now suppose that $(K 2)$ holds. Let us evaluate the moment generating function of $V_{n}^{*}(f)$ :

$$
\phi_{V_{n}^{*}(f)}(t)=\prod_{j=1}^{k} \phi_{\left(E_{j}-1\right)}\left(t f(j) j^{-1} \sigma_{n}(f)^{-1}\right)
$$

Recall that, in this case, $\sigma_{n}(f) \uparrow \infty$. It follows from $(K 2)$, that for any $u_{0}>0$ for a fixed $t$, for $k$ large enough,

$$
\left|t f(j) j^{-1} \sigma_{n}(f)^{-1}\right| \leq u_{0}
$$


uniformly in $j \geq 1$. At this step, we use the expansion of $\phi_{\left(E_{j}-1\right)}$ in the neighborhood of zero :

$$
\psi_{\left(E_{j}-1\right)}(u)=1+u^{2} / 2+u^{3} g(u)
$$

where there exists $u_{0}$ such that

$$
0 \leq u \leq u_{0} \Rightarrow|g(u)| \leq 1
$$

Using the uniform bound in (6.7), we get

$$
\left.\left.\phi_{\left(E_{j}-1\right)}\left(t f(j) j^{-1} \sigma_{n}(f)^{-1}\right)=1+\frac{1}{2}\left(t f(j) j^{-1} \sigma_{n}(f)\right)^{-1}\right)^{2}+\left(t f(j) j^{-1} \sigma_{n}(f)\right)^{-1}\right)^{3} g_{0, j, n}(t),
$$

where $\left|g_{0, j, n}(t)\right| \leq 1$ for all $1 \leq j \leq k$. By the uniform boundedness of the error term, we have $\left.\left.\left.\left.\log \phi_{\left(E_{j}-1\right)}\left(t f(j) j^{-1} \sigma_{n}(f)\right)^{-1}\right)=\frac{1}{2}\left(t f(j) j^{-1} \sigma_{n}(f)\right)^{-1}\right)^{2}+\left(t f(j) j^{-1} \sigma_{n}(f)\right)^{-1}\right)^{3} g_{0, j, n}(t)+\left(t f(j) j^{-1} \sigma_{n}(f)\right)^{-1}\right)^{3} g_{1, j, n}(t)$,

where, always $\left|g_{1, j, n}(t)\right| \leq 1$ for all $1 \leq j \leq k$. Finally

$$
\phi_{V_{n}^{*}(f)}(t)=\exp \left(\sum_{j=1}^{k} \log \phi_{\left(E_{j}-1\right)}\left(t f(j) j^{-1} \sigma_{n}(f)\right)^{-1}\right)=\exp \left(t^{2} / 2+g_{2, j, n}(t) \times t^{3} \sigma_{n}(f)^{-3} \sum_{j=1}^{k} f(j)^{3} j^{-3}\right)
$$

with $\left|g_{1, j, n}(t)\right| \leq 2$ for all $1 \leq j \leq k$. Since

$$
\left.0 \leq \sigma_{n}(f)^{-3} \sum_{j=1}^{k} f(j)^{3} j^{-3}\right) \leq B(n, f) \times \sigma_{n}(f)^{-2} \sum_{j=1}^{k} f(j)^{2} j^{-2}=B(n, f) \rightarrow 0 .
$$

Hence

$$
\phi_{V_{n}^{*}(f)}(t) \rightarrow \exp \left(t^{2} / 2\right)
$$

and

$$
V_{n}^{*}(f) \rightarrow \mathcal{N}(0,1)
$$

Lemma 2 Let $(a, b) \in \mathbb{R}^{2}$ and suppose that for any couple $\left(f_{1}, f_{2}\right) \in \mathcal{F}_{h}^{2}, h=1,2$,

$$
\lim _{n \rightarrow \infty} \frac{1}{\sigma_{n}\left(f_{1}\right) \sigma_{n}\left(f_{2}\right)} \sum_{j=1}^{k} f_{1}(j) f_{2}(j) j^{-2}=\Gamma\left(f_{1}, f_{2}\right) \text { exists. }
$$

Then for $h=1, a V_{n}^{*}\left(f_{1}\right)+b V_{n}^{*}\left(f_{2}\right)$ weakly converges to

$$
a \mathcal{L}\left(f_{1}\right)+b \mathcal{L}\left(f_{2}\right)
$$

and for $h=2, a V_{n}^{*}\left(f_{1}\right)+b V_{n}^{*}\left(f_{2}\right)$ weakly converges to a normal random of variance :

$$
v\left(a, b, f_{1}, f_{2}\right)=a^{2}+b^{2}+2 a b \Gamma_{1}\left(f_{1}, f_{2}\right)
$$

In both cases, the finite-distributions of $\left\{V_{n}^{*}(f), f \in \mathcal{F}_{h}\right\}$ weakly converge to those of the process $\mathcal{L}$ for $h=1$ and, for $h=2$, to those of a Gaussian process of covariance function

$$
\Gamma\left(f_{1}, f_{2}\right)=\lim _{n \rightarrow \infty} \frac{1}{\sigma_{n}\left(f_{1}\right) \sigma_{n}\left(f_{2}\right)} \sum_{j=1}^{k} f_{1}(j) f_{2}(j) j^{-2}
$$

provided these numbers are finite.

Proof. The case $h=1$ is straightforward. For $h=2$, we slightly change the proof of the previous lemma from (6.6). Put $V_{n}\left(a, b, f_{1}, f_{2}\right)=a V_{n}^{*}\left(f_{1}\right)+b V_{n}^{*}\left(f_{2}\right)$ and

$$
c(n, j)=\sigma_{n}\left(f_{1}\right)^{-1} a f_{1}(j) j^{-1}+\sigma_{n}\left(f_{2}\right)^{-1} b f_{2}(j) j^{-1} .
$$


We always have, for $\left(f_{1}, f_{2}\right) \in \mathcal{F}_{2}^{2}$,

$$
\sup _{j}|c(n, j)| \rightarrow 0
$$

and

$$
\sum_{j=1}^{k} c(n, j)^{2}=a^{2}+b^{2}+2 a b \frac{1}{\sigma_{n}\left(f_{1}\right) \sigma_{n}\left(f_{2}\right)} \sum_{j=1}^{k} f_{1}(j) f_{2}(j) j^{-2} \rightarrow v\left(a, b, f_{1}, f_{2}\right)=a^{2}+b^{2}+2 a b \Gamma\left(f_{1}, f_{2}\right) .
$$

By using the same arguments in (6.6) and (6.8), we have

$$
\phi_{V_{n}\left(a, b, f_{1}, f_{2}\right)}(t)=\exp \left(\sum_{j=1}^{k} \log \psi_{\left(E_{j}-1\right)}(t c(n, j))=\exp \left(\left\{\sum_{j=1}^{k} c(n, j)^{2}\right\} t^{2} / 2+g_{3, j, n}(t) \times t^{3}\left(\sum_{j=1}^{k} c(n, j)^{3}\right)\right),\right.
$$

with $\left|g_{3, j, n}(t)\right| \leq 2$ for all $1 \leq j \leq k$. Since

$$
0 \leq \sum_{j=1}^{k} c(n, j)^{3} \leq\left\{a B\left(n, f_{1}\right)+b B\left(n, f_{2}\right)\right\} \times \sum_{j=1}^{k} c(n, j)^{2} \rightarrow 0,
$$

we get

$$
\phi_{V_{n}\left(a, b, f_{1}, f_{2}\right)}(t) \rightarrow \exp \left(v\left(a, b, f_{1}, f_{2}\right) t^{2} / 2\right) .
$$

This achieves the proof. Now, these methods are reproducible for any finite linear combination

$$
a_{1} V_{n}^{*}\left(f_{1}\right)+a_{2} V_{n}^{*}\left(f_{2}\right)+\ldots+a_{m} V_{n}^{*}\left(f_{m}\right) .
$$

In all cases, we find the same finite distribution laws. For $h=1,\left(V_{n}^{*}\left(f_{1}\right), \ldots, V_{n}\left(f_{m}\right)\right)$ converges in law to $\left(\mathcal{L}\left(f_{1}\right)\right.$, $\left.\ldots, \mathcal{L}\left(f_{m}\right)\right)$ and for $h=2,\left(V_{n}^{*}\left(f_{1}\right), \ldots, V_{n}^{*}\left(f_{m}\right)\right)$ converges to a Gaussian vector of variance-covariance matrix $\left(\Gamma\left(f_{i}, f_{j}\right), 1 \leq i, j \leq m\right)$ provided these numbers are finite.

\section{Appendix}

7.1 Check Hypothesis (L4) for $\tau>1 / 2$

We shall consider $\mathcal{F}_{2}(a, b)$ as a normed vector by identifying $f_{\tau}(j)=j^{\tau}$ with $\tau$ and setting $f_{\tau_{1}}+f_{\tau_{2}}=f_{\tau_{1}+\tau_{2}}$ and $f_{\tau_{1}} \leq f_{\tau_{2}}$ iff $\tau_{1} \leq \tau_{2}$. We put without lost of generality that $b-a=1$ and that $\varepsilon^{2}=1 / p$. Next we divide $\mathcal{F}_{2}(a, b)$ into $p$ intervals $\left[0, \tau_{1}\left[,\left[\tau_{1}, \tau_{2}\left[, \ldots,\left[\tau_{p-1}, \tau_{p}\right]\right.\right.\right.\right.$. Now, put

$$
\sigma_{n}^{2}(1, \tau)=k^{-2 \tau+1} \sigma_{n}^{2}\left(f_{\tau}\right)
$$

and consider $\tau_{i}<v<\tau<\tau_{i+1}$ and let $i=1$ for short, $\tau-v=\delta$ and

$$
\begin{gathered}
d_{n}^{2}\left(f_{v}, f_{\tau}, j\right)=\left(f_{v}(j) / \sigma_{n}\left(f_{v}\right)-f_{\tau}(j) / \sigma_{n}\left(f_{\tau}\right)\right)^{2} j^{-2}\left(E_{j}-1\right)^{2} \\
\left\{\frac{k^{-2 v+1}}{\sigma_{n}^{2}(1, v)} j^{2 v-2}+\frac{k^{-2 \tau+1}}{\sigma_{n}^{2}(1, v)} j^{2 \tau-2}-2 \frac{k^{-v-\tau+1}}{\sigma_{n}(1, v) \sigma_{n}(1, \tau)} j^{\tau+v-2}\right\}\left(E_{j}-1\right)^{2} \\
=\left\{T_{0}(n, \tau, j)+T_{0}(n, v, j)-2 T_{1}(n, v, \tau)\right\}\left(E_{j}-1\right)^{2} .
\end{gathered}
$$

Let us handle $T_{0}(n, \tau, j)$. By adding to it this null term

$$
0=\left\{-\sigma_{n}^{-2}(1, v) k^{-2 \tau+1} j^{2 \tau-2}-\sigma_{n}^{-2}(1, v) k^{-2 \tau+1} j^{2 \tau-2}\right\}+\left\{-\sigma_{n}^{-2}(1, v) k^{-2 v+1} j^{2 v-2}+-\sigma_{n}^{-2}(1, v) k^{-2 v+1} j^{2 v-2}\right\},
$$

we get

$$
\begin{gathered}
T_{0}(n, \tau, j)=\left(\frac{1}{\sigma_{n}^{2}(1, \tau)}-\frac{1}{\sigma_{n}^{2}(1, v)}\right) k^{-2 \tau+1} j^{2 \tau-2} \\
+\frac{k^{-2 v+1}}{\sigma_{n}^{2}(1, v)} j^{2 v-2}\left(1-(j / k)^{2 \delta}\right)+\frac{k^{-2 v+1}}{\sigma_{n}^{2}(1, v)} j^{2 v-2}
\end{gathered}
$$

where we used in the previous line (7.2) the following identity

$$
k^{-2 \tau+1} j^{2 \tau-2}=k^{-2 v+1} j^{2 v-2}+k^{-2 v+1} j^{2 v-2}\left(1-(j / k)^{2 \delta}\right) .
$$


Using again (7.4) in (7.2), we arrive at

$$
\begin{gathered}
T_{0}(n, \tau, j) \\
=T_{0}(n, v, j)+\left(\frac{1}{\sigma_{n}^{2}(1, \tau)}-\frac{1}{\sigma_{n}^{2}(1, v)}\right) k^{-2 v+1} j^{2 v-2} \\
+\left(\frac{1}{\sigma_{n}^{2}(1, \tau)}-\frac{1}{\sigma_{n}^{2}(1, v)}\right) k^{-2 v+1} j^{2 v-2}\left(1-(j / k)^{2 \delta}\right)+\frac{k^{-2 v+1}}{\sigma_{n}^{2}(1, v)} j^{2 v-2}\left(1-(j / k)^{2 \delta}\right) .
\end{gathered}
$$

We use the same techniques to also get

$$
\begin{gathered}
T_{1}(n, v, \tau) \\
=T_{0}(n, v, j)+\left(\frac{1}{\sigma_{n}(1, \tau) \sigma_{n}(1, v)}-\frac{1}{\sigma_{n}^{2}(1, v)}\right) k^{-2 v+1} j^{2 v-2} \\
+\left(\frac{1}{\sigma_{n}(1, \tau) \sigma_{n}(1, v)}-\frac{1}{\sigma_{n}^{2}(1, v)}\right) k^{-2 v+1} j^{2 v-2}\left(1-(j / k)^{\delta}\right)+\frac{k^{-2 v+1}}{\sigma_{n}^{2}(1, v)} j^{2 v-2}\left(1-(j / k)^{\delta}\right)
\end{gathered}
$$

Let us handle the $\sigma_{n}^{-2}(1, \tau)-\sigma_{n}^{-2}(1, v)$ in (7.1). We get, by using the same methods,

$$
\sigma_{n}^{-2}(1, \tau)-\sigma_{n}^{-2}(1, v)=\sigma_{n}^{-2}(1, \tau) \sigma_{n}^{-2}(1, \tau) k^{-2 v+1} \sum_{j=1}^{k} j^{2 v-2}\left(1-(j / k)^{2 \delta}\right) .
$$

We already noticed that $\sigma_{n}^{2}(1, \tau) \rightarrow(2 \tau-1)^{-1} \in\left[B=(2 b-1)^{-1}, A=(2 a-1)^{-1}\right]$ uniformly in $\tau \in[a, b]$ by a continuity convergence argument. For $\varepsilon$ chosen such that $2 a-1-2 \varepsilon>0$, we get

$$
k^{-2 v+1} \sum_{j=1}^{k} j^{2 v-2}\left(1-(j / k)^{2 \delta}\right) \leq \sum_{j=1}^{k} j^{-1}(j / k)^{2 a-1}\left(1-(j / k)^{2 \varepsilon^{2}}\right)=k^{2 a-1} \sum_{j=1}^{k} j^{2 a-2}-k^{2 \varepsilon^{2}} j^{2 a-2+2 \varepsilon^{2}} .
$$

By using the asymptotic approximations to the corresponding integrals, we get, as $n \rightarrow \infty$,

$$
\begin{gathered}
k^{2 a-1} \sum_{j=1}^{k} j^{2 a-2}-k^{2 \varepsilon^{2}} j^{2 a-2+2 \varepsilon^{2}}=k^{-2 a+1} K\left\{(1+o(1)) \frac{k^{2 a-1}-1}{2 a-1}\right\}-\left(1+o(1) k^{-2 \varepsilon^{2}} \frac{k^{2 a-1+2 \varepsilon^{2}}}{2 a-1+2 \varepsilon^{2}}\right) \\
\rightarrow\left\{(2 a-1)^{-1}-\left(2 a-1+2 \varepsilon^{2}\right)^{-1}\right\} \leq\left(2 \varepsilon^{2}\right) /(2 a-1)^{2} .
\end{gathered}
$$

We conclude that, for large value of $n$,

$$
k^{-2 v+1} \sum_{j=1}^{k} j^{2 v-2}\left(1-(j / k)^{2 \delta}\right) \leq K \varepsilon^{2}
$$

and hence

$$
\left|\sigma_{n}^{-2}(1, \tau)-\sigma_{n}^{-2}\right| \leq K \varepsilon^{2}
$$

for some positive universal constant $K$. This constant is generic and may change from line to line. In the same spirit, we have, for this term in (7.1),

$$
\left\{\sigma_{n}(1, \tau) \sigma_{n}(1, v)\right\}^{-1}-\sigma_{n}^{-2}(1, v)=\sigma_{n}(1, v)^{-1} \sigma_{n}(1, \tau)^{-1}-\sigma_{n}^{-1}(1, v)=-\frac{\sigma_{n}^{-2}(1, \tau)-\sigma_{n}^{-2}(1, v)}{\sigma_{n}(1, v)^{2} \sigma_{n}(1, \tau)\left(\sigma_{n}(1, \tau)+\sigma_{n}(1, v)\right)}
$$

The methods used above lead to

$$
\left|\left\{\sigma_{n}(1, \tau) \sigma_{n}(1, v)\right\}^{-1}-\sigma_{n}^{-2}(1, v)\right| \leq K \varepsilon^{2}
$$

Now returning to Formulae (7.1), (7.2) and (7.1), we see that the terms $T_{0}(n, v, j)$ disappears as $T_{0}(n, v, j)-$ $2 T_{0}(n, v, j)+T_{0}(n, v, j)=0$. Computing the expectation of the remainder terms, we get

$$
E d_{n}^{2}\left(f_{v}, f_{\tau}, j\right) \leq 2\left|\frac{1}{\sigma_{n}^{2}(1, \tau)}-\frac{1}{\sigma_{n}^{2}(1, v)}\right| j^{-1}(j / k)^{2 a-1}
$$




$$
+2\left|\frac{1}{\sigma_{n}(1, \tau) \sigma_{n}(1, v)}-\frac{1}{\sigma_{n}^{2}(1, v)}\right| j^{-1}(j / k)^{2 a-1}+2 j^{-1}(j / k)^{2 a-1}\left(1-(j / k)^{2 \delta}\right) .
$$

The left-member does not depend on $(\nu, \tau) \in\left[\tau_{i-1}, \tau_{i}\right]$ so that, with the application (7.7) and (7.8), we obtain

$$
\max _{(\nu, \tau) \in\left[\tau_{i-1}, \tau_{i}\right]} d_{n}^{2}\left(f_{v}, f_{\tau}, j\right) \leq K\left\{(j / k)^{2 a-1} \varepsilon^{2}+2 j^{-1}(j / k)^{2 a-1}\left(1-(j / k)^{2 \varepsilon^{2}}\right)\right\}\left(E_{j}-1\right)^{2} .
$$

Finally, by (7.7),

$$
\sum_{j=1}^{k} \mathbb{E} \sup _{(\nu, \tau) \in\left[\tau_{i-1}, \tau_{i}\right]} d_{n}^{2}\left(f_{v}, f_{\tau}, j\right) \leq K \varepsilon^{2},
$$

for large values of $n$. Then

$$
\log N_{[\mathrm{]}}\left(\epsilon, \mathcal{F}_{h}, L_{2}^{n}\right) \leq p \leq K \varepsilon^{-2}
$$

And this ensures $(L 4)$ of Theorem 4.

\subsection{Continuous Convergence}

In order to prove (5.2), suppose for each $N>0$, there exists a value $n \geq N$ and a couple $\left(f_{1}, f_{2}\right)$ such that

$$
\rho_{n}^{2}\left(f_{1}, f_{2}\right)<\left(1-\Gamma\left(f_{1}, f_{2}\right)\right)
$$

We may lessen the notations and put $\rho_{n}^{2}\left(f_{1}, f_{2}\right)=\rho_{n}^{2}\left(\tau_{1}, \tau_{2}\right)$ for $f_{1}(j)=j^{\tau_{1}}$ and $f_{2}(j)=j^{\tau_{2}} \in(a, b)^{2}$. It is easy to prove that

$$
\rho_{n}^{2}\left(\tau_{1}, \tau_{2}\right) \rightarrow 2\left(1-\Gamma\left(\tau_{1}, \tau_{2}\right)\right)
$$

continuously, that is,

$$
\rho_{n}^{2}\left(\tau_{1, n}, \tau_{2, n}\right) \rightarrow 2\left(1-\Gamma\left(\tau_{1}, \tau_{2}\right)\right) .
$$

if $\left(\tau_{1, n}, \tau_{2, n}\right) \rightarrow\left(\tau_{1}, \tau_{2}\right)$, as $n \rightarrow+\infty$. But, with our hypothesis $(\mathrm{H})$, we can find a sequence of integers $n_{1}<n_{2}<$ $\ldots<n_{k}<n_{k+1}<.$. such that and a sequence of couples $\left(\tau_{1, n_{k}}, \tau_{2, n_{k}}\right) \in(a, b)^{2}, k=1,2, \ldots$ such that for any $k$,

$$
\rho_{n}^{2}\left(\tau_{1, n_{k}}, \tau_{2, n_{k}}\right)<\left(1-\Gamma\left(\tau_{1, n_{k}}, \tau_{2, n_{k}}\right) .\right.
$$

By the Bolzano-Weierstrass Theorem, we may extract from $\left(\tau_{1, n_{k}}, \tau_{2, n_{k}}\right)$ a subsequence, denoted $\left(\tau_{1, n_{k}^{*}}, \tau_{2, n_{k}^{*}}\right)$ converging to some $\left(\tau_{1}, \tau_{2}\right) \in(a, b)^{2}$ and by the continuity result

$$
\rho_{n^{*}}^{2}\left(\tau_{1, n_{k}^{*}}, \tau_{2, n_{k}^{*}}\right) \rightarrow 2\left(1-\Gamma\left(\tau_{1}, \tau_{2}\right)\right) .
$$

This violates (H1) and then proves (5.2).

\section{Acknowledgements}

This paper was partly written in University of Orleans (France) while invited by MAPMO and in University of Briskra (Algeria). Thanks to our guests for kind hospitality.

\section{Reference}

Beirlant, J., Goegebeur, Y., \& Teugels, J. (2004). Statistics of Extremes Theory and Applications. Wiley. http://dx.doi.org/10.1002/0470012382

Csörgö, M., Csörgö, S., Horvàth, L., \& Mason, M. (1986). Weighted empirical and quantile processes. Ann. Probab., 14, 31-85. http://dx.doi.org/10.1214/aop/1176992617

Csörgö, S., Deheuvels, P., \& Mason, D. M. (1985). Kernel estimates of the tail index of a distribution. Ann. Statist., 13, 1050-1077. http://dx.doi.org/10.1214/aos/1176349656

Csörgö, S., \& Mason, D. M. (1985). Central limit theorem for sums of extreme values. Math. Proc. Cambridge philos. Soc., 98, 547-558. http://dx.doi.org/10.1017/S0305004100063751

De Haan, L. (1970). On regular variation and its application to the weak convergence of sample extremes. Mathematical Centre Tracts, 32, Amsterdam.

De Haan, L., \& Feireira A. (2006). Extreme value theory: An introduction. Springer. 
De Haan, L., \& Resnick, S. I. (1980). A simple asymptotic estimate for the index of a stable distribution. J. Roy. Statist. Soc., 42B, 83-87.

Dekkers, A. L. M., Einmahl, J. H. J., \& De Haan, L. (1989). A moment estimator for the index of an extreme value distribution. Ann. Statist., 17(4), 1833-1855.

Dème, E., LO, G. S., \& Diop, A. (2010). On the generalized Hill process for small parameters and applications. J. Stat. Theory Appl., 11(4). Retrieved from http://arxiv.org/pdf/1111.4564

Diop, A., \& Lo, G. S. (2006). Generalized Hill's Estimator. Far East J. Theor. Statist., 20(2), 129-149.

Diop, A., \& Lo, G. S. (2009). Ratio of Generalized Hill's Estimator and its asymptotic normality theory. Math. Method. Statist., 18(2), 117-133.

Galambos, J. (1985). The Asymptotic Theory of Extreme Order Statistics. New York: Wiley.

Groeneboom Lopuha, H. P., \& Wolf, P. P. (2003). Kernel-Type Estimator for the extreme Values index. Ann. Statist., 31(6), 1956-1995.

Hall, P. (1978) Representation and limit theorem for extreme value distributions. J. Appl. Probab., 15, 639-644.

Hall, P. (1982). On simple estimates of an exponent of regular variation. J. Roy. Statist. Soc., Ser. B, 44, 37-42.

Hasofer, A. M., \& Wang, Z. (1992). A test for extreme value domain of attraction. J. Amer. Statist. Assoc., 87(417), 171-177.

Hauesler, E., \& Teugels, L. (1985). On asymptotic normality of Hill's estimator for the exponent of regular variation. Ann. Statist., 13, 743-756.

Hill, B. M. (1975). A simple general approach about the inference about the tail index of a distribution. Ann. Statist., 3, 1163-1174.

Lo, G. S. (1989). A note on the asymptotic normality of sums of extreme values. J. Statist. Plan. and Inf., 22, 89-94.

Pickands, J. (1975). Statistical Inference using extreme order statistics. Ann. Statist., 3, 119-131.

Resnick, S. I. (1987). Extreme Values, Regular Variation and Point Processes. New York: Springer-Verbag.

Shorack, G. R., \& Wellner, J. A. (1986). Empirical Processes with Applications to Statistics. New York: WileyInterscience.

van der Vaart, A. W., \& Wellner, J. A. (1996). Weak Convergence and Empirical Processes. With Applications to Statistics. New York: Springer. 